\title{
Correction to: Expanding the genetic heterogeneity of intellectual disability
}

\author{
Shams Anazi ${ }^{1}$ - Sateesh Maddirevula ${ }^{1}$. Vincenzo Salpietro ${ }^{21} \cdot$ Yasmine T. Asi $^{2} \cdot$ Saud Alsahli $^{1}$. Amal Alhashem ${ }^{3}$. \\ Hanan E. Shamseldin ${ }^{1}$ • Fatema AlZahrani ${ }^{1}$. Nisha Patel ${ }^{1}$ • Niema Ibrahim ${ }^{1}$ • Firdous M. Abdulwahab ${ }^{1}$. \\ Mais Hashem ${ }^{1}$. Nadia Alhashmi ${ }^{4}$. Fathiya Al Murshedi ${ }^{4}$ Adila Al Kindy ${ }^{4}$ Ahmad Alshaer ${ }^{11}$ - Ahmed Rumayyan ${ }^{5,6}$. \\ Saeed AI Tala ${ }^{7}$. Wesam Kurdi ${ }^{8}$. Abdulaziz Alsaman ${ }^{16}$. Ali Alasmari ${ }^{16}$. Selina Banu ${ }^{22}$. Tipu Sultan ${ }^{23}$. \\ Mohammed M. Saleh ${ }^{16} \cdot$ Hisham Alkuraya ${ }^{9} \cdot$ Mustafa A. Salih $^{10} \cdot$ Hesham Aldhalaan ${ }^{11} \cdot$ Tawfeg Ben-Omran $^{12}$. \\ Fatima AI Musafri ${ }^{12} \cdot$ Rehab Ali $^{12}$. Jehan Suleiman ${ }^{13} \cdot$ Brahim Tabarki $^{3} \cdot$ Ayman W. El-Hattab $^{14} \cdot$ Caleb Bupp $^{17}$. \\ Majid Alfadhel $^{18} \cdot$ Nada Al Tassan $^{1,15} \cdot$ Dorota Monies $^{1,15} \cdot$ Stefan T. Arold ${ }^{19} \cdot$ Mohamed Abouelhoda $^{1,15}$. \\ Tammaryn Lashley ${ }^{2} \cdot$ Henry Houlden ${ }^{21} \cdot$ Eissa Faqeih $^{16} \cdot$ Fowzan S. Alkuraya $^{1,3,15,20,17 \text { (D) }}$
}

Published online: 29 December 2017

c) Springer-Verlag GmbH Germany, part of Springer Nature 2017

\section{Correction to: Hum Genet (2017) 136:1419-1429 https://doi.org/10.1007/s00439-017-1843-2}

Variant nomenclature discrepancy was identified in the article "Expanding the genetic heterogeneity of intellectual disability", Human Genetics, November 2017, Volume 136, Issue 11-12, pp 1419-1429 after its publication. Specifically, the nomenclature of GTF3C3 was originally listed as NM_012086.4:c.1382+3A>G when it should be NM_012086.4:c.1390+3A>G, the nomenclature of $M A D D$ was originally listed as NM_001135943.1:c.2930T>G:p.(Val977Gly) when it should be NM_001135943.1:c.2930T>G:p.(Leu977Arg), and the variant NM_001164416.1:c.124C >T:p.(Arg42*) was listed under the gene's name $V W A 3 B$ when it should be $H 2 B F M$. The following changes were made in the attached corrections:
1. Nomenclature of $G T F 3 C 3$ is changed to NM_012086.4:c.1390+3A $>$ G in 15DG0315 (from NM_012086.4:c.1382+3A $>$ G) in the main text, Table S1, Table S2 and Figure S1.

2. The variant NM_001164416.1:c.124C > T:p.(Arg42*) in 17DG0782 is now correctly listed under the name H2BFM in the abstract, Table 1, Table S1 and Table S2.

3. Nomenclature of $M A D D$ is changed to NM_001135943.1:c.2930T>G:p.(Leu977Arg) in 17DG0771 (From NM_001135943.1:c.2930T>G:p. (Val977Gly) in results, Table 1, Table S1, and Table S2.

The authors sincerely apologize for these errors and appreciate the opportunity to mend the records.

The original article can be found online at https://doi.org/10.1007/ s00439-017-1843-2.

Electronic supplementary material The online version of this article (https://doi.org/10.1007/s00439-017-1859-7) contains supplementary material, which is available to authorized users.

Fowzan S. Alkuraya falkuraya@kfshrc.edu.sa

Extended author information available on the last page of the article 


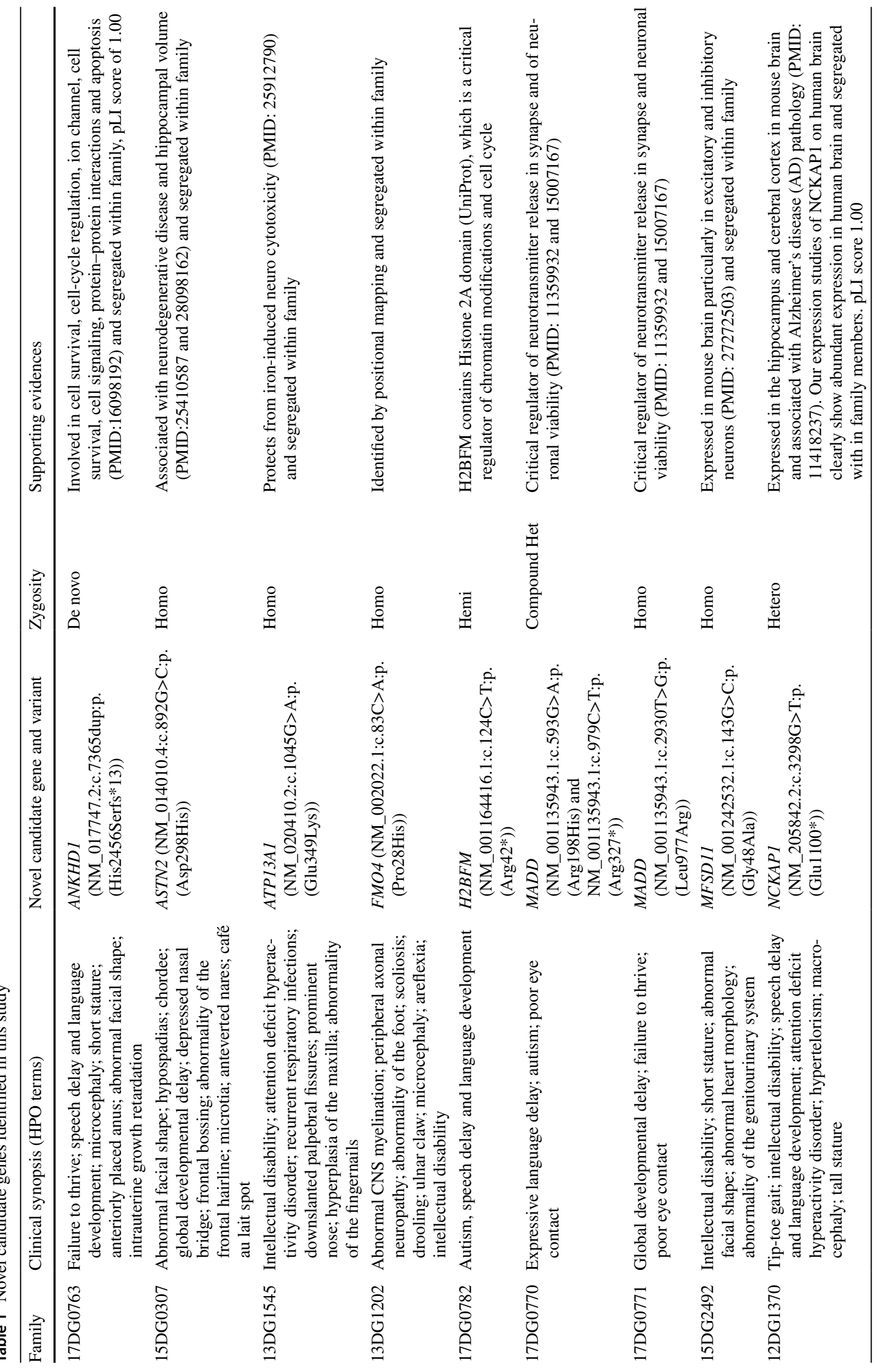




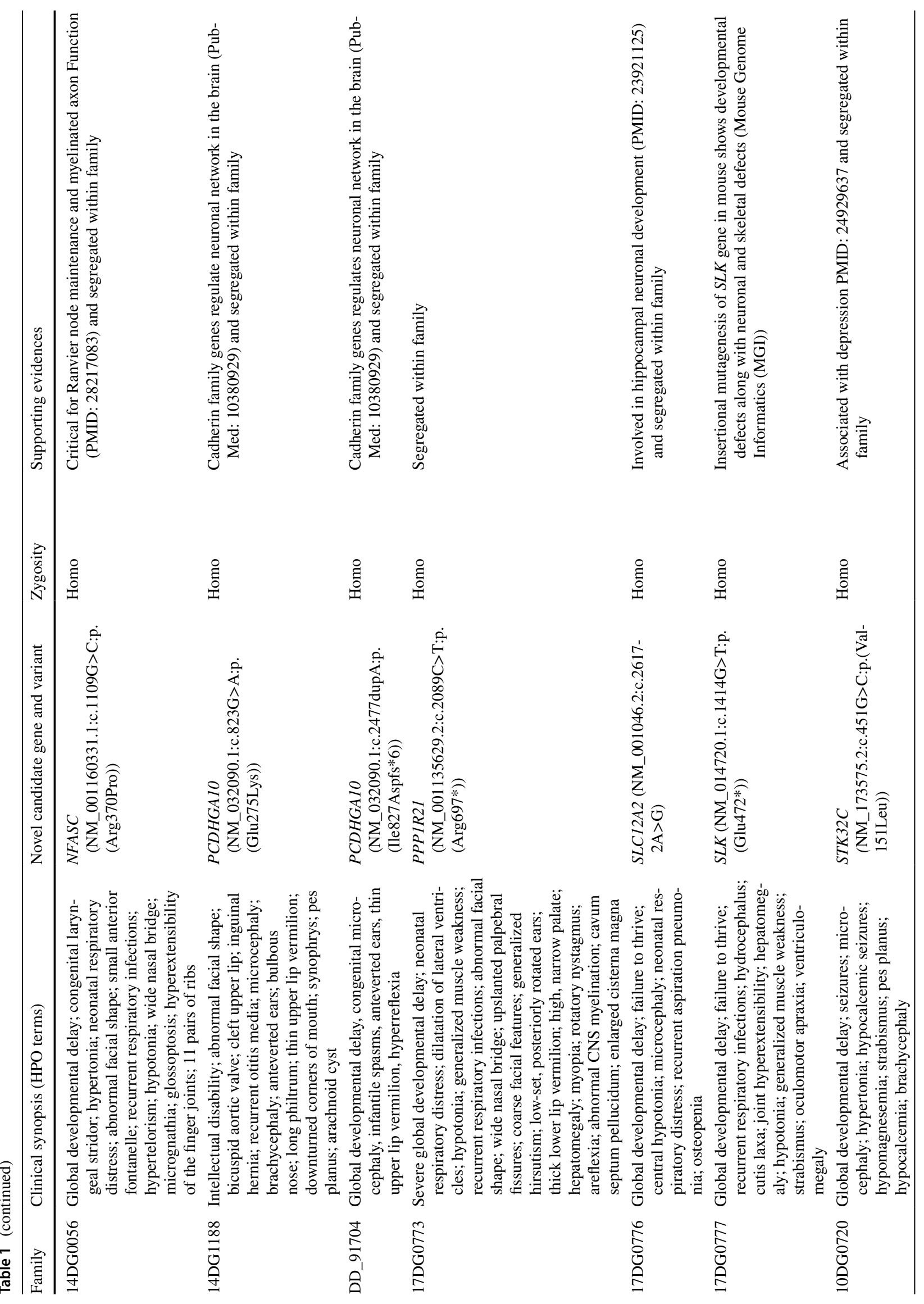




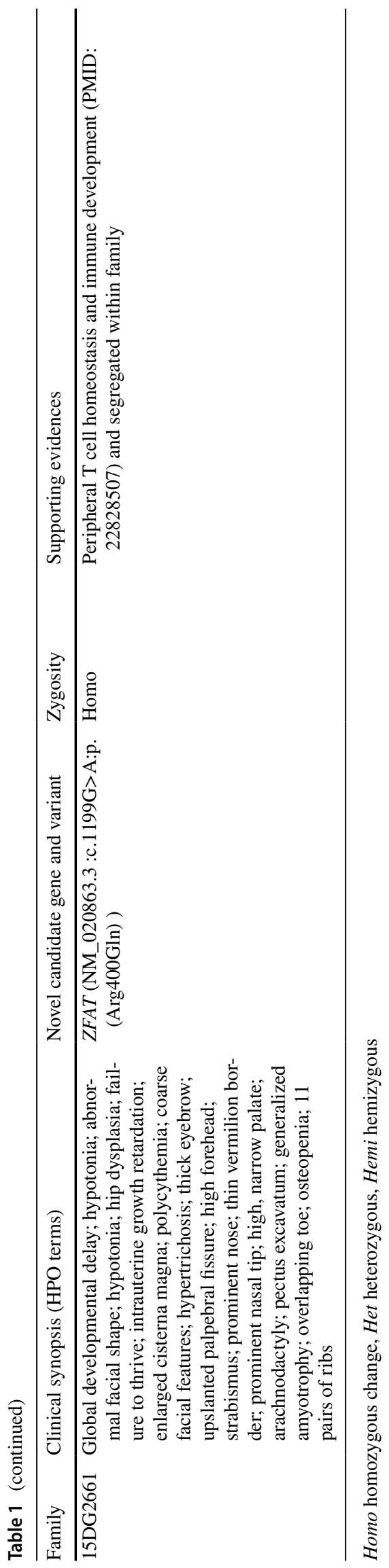




\section{Affiliations}

Shams Anazi ${ }^{1}$ - Sateesh Maddirevula ${ }^{1}$. Vincenzo Salpietro ${ }^{21} \cdot$ Yasmine T. Asi $^{2} \cdot$ Saud Alsahli $^{1} \cdot$ Amal Alhashem $^{3}$. Hanan E. Shamseldin ${ }^{1}$ • Fatema AlZahrani ${ }^{1}$. Nisha Patel ${ }^{1}$ • Niema Ibrahim ${ }^{1}$. Firdous M. Abdulwahab ${ }^{1}$.

Mais Hashem ${ }^{1}$. Nadia Alhashmi ${ }^{4}$. Fathiya Al Murshedi ${ }^{4}$. Adila Al Kindy ${ }^{4}$. Ahmad Alshaer ${ }^{11}$ • Ahmed Rumayyan ${ }^{5,6}$. Saeed AI Tala ${ }^{7}$. Wesam Kurdi ${ }^{8}$. Abdulaziz Alsaman ${ }^{16}$. Ali Alasmari ${ }^{16}$. Selina Banu ${ }^{22}$. Tipu Sultan ${ }^{23}$. Mohammed M. Saleh ${ }^{16}$. Hisham Alkuraya ${ }^{9} \cdot$ Mustafa A. Salih $^{10} \cdot$ Hesham Aldhalaan $^{11} \cdot$ Tawfeg Ben-Omran $^{12}$. Fatima Al Musafri ${ }^{12} \cdot$ Rehab Ali $^{12}$. Jehan Suleiman ${ }^{13} \cdot$ Brahim Tabarki $^{3}$. Ayman W. El-Hattab ${ }^{14}$. Caleb Bupp ${ }^{17}$. Majid Alfadhel $^{18} \cdot$ Nada Al Tassan $^{1,15} \cdot$ Dorota Monies $^{1,15} \cdot$ Stefan T. Arold ${ }^{19} \cdot$ Mohamed Abouelhoda $^{1,15}$. Tammaryn Lashley $^{2} \cdot$ Henry Houlden ${ }^{21} \cdot$ Eissa Faqeih $^{16} \cdot$ Fowzan S. Alkuraya $^{1,3,15,20,17 \mathbb{C}}$

1 Department of Genetics,

King Faisal Specialist Hospital and Research Center, Riyadh, Saudi Arabia

2 Queen Square Brain Bank for Neurological Disorders, Department of Molecular Neuroscience, UCL Institute of Neurology, University College London, London, UK

3 Department of Pediatrics, Prince Sultan Military Medical City, Riyadh, Saudi Arabia

4 Department of Genetics, College of Medicine, Sultan Qaboos University, Muscat, Sultanate of Oman

5 King Saud bin Abdulaziz University for Health Sciences, Riyadh, Saudi Arabia

6 Neurology Division, Department of Pediatrics, King Abdulaziz Medical City, Riyadh, Saudi Arabia

7 Department of Pediatrics and Genetic Unit, Armed Forces Hospital Khamis Mushayt, Riyadh, Saudi Arabia

8 Department of Obstetrics and Gynecology, King Faisal Specialist Hospital, Riyadh, Saudi Arabia

9 Department of Ophthalmology, Specialized Medical Center Hospital, Riyadh, Saudi Arabia

10 Division of Pediatric Neurology, Department of Pediatrics, King Khalid University Hospital and College of Medicine, King Saud University, Riyadh, Saudi Arabia

11 Pediatric Neurology, King Faisal Specialist Hospital and Research Center, Riyadh, Saudi Arabia

12 Clinical and Metabolic Genetics, Department of Pediatrics, Hamad Medical Corporation, Doha, Qatar

13 Division of Neurology, Department of Pediatrics, Tawam Hospital, Al Ain, United Arab Emirates
14 Division of Clinical Genetics and Metabolic Disorders, Department of Pediatrics, Tawam Hospital, Al-Ain, United Arab Emirates

15 Saudi Human Genome Program, King Abdulaziz City for Science and Technology, Riyadh, Saudi Arabia

16 Department of Pediatric Subspecialties, Children's Hospital, King Fahad Medical City, Riyadh, Saudi Arabia

17 Spectrum Health Genetics, Grand Rapids, MI, USA

18 Genetics Division, Department of Pediatrics, King Abdullah International Medical Research Centre, King Saud bin Abdulaziz University for Health Sciences, King Abdulaziz Medical City, Riyadh, Saudi Arabia

19 Division of Biological and Environmental Sciences and Engineering (BESE), Computational Bioscience Research Center (CBRC), King Abdullah University of Science and Technology (KAUST), Thuwal 23955-6900, Saudi Arabia

20 Division of Biological and Environmental Sciences and Engineering (BESE), King Abdullah University of Science and Technology (KAUST), Thuwal 23955-6900, Saudi Arabia

21 Department of Molecular Neuroscience, UCL Institute of Neurology, London, UK

22 Department of Pediatric Neurology, ICH and SSF Hospital Mirpur, Dhaka 1216, Bangladesh

23 Department of Pediatric Neurology, Institute of Child Health and The Children's Hospital Lahore, 381-D/2, Lahore, Pakistan 